\title{
Effect of Adaptive and Cooperative Adaptive Cruise Control on Throughput of Signalized Arterials
}

\author{
Armin Askari, Daniel Albarnaz Farias, Alex A. Kurzhanskiy, Pravin Varaiya *
}

August 13, 2018

\begin{abstract}
The paper evaluates the influence of the maximum vehicle acceleration and variable proportions of $\mathrm{ACC} / \mathrm{CACC}$ vehicles on the throughput of an intersection. Two cases are studied: (1) free road downstream of the intersection; and (2) red light at some distance downstream of the intersection. Simulation of a 4-mile stretch of an arterial with 13 signalized intersections is used to evaluate the impact of $(\mathrm{C}) \mathrm{ACC}$ vehicles on the mean and standard deviation of travel time as the proportion of (C)ACC vehicles is increased. The results suggest a very high urban mobility benefit of (C)ACC vehicles at little or no cost in infrastructure.
\end{abstract}

\section{Introduction}

The capacity of a freeway will increase if vehicles traveling at the speed limit could do so at much shorter headway, since the flow is just the product of speed and density, which is just the inverse of the headway. Adaptive cruise control (ACC) and cooperative adaptive cruise control (CACC) are longitudinal control technologies that permit headway reduction by a factor of 2 or 3 (compared with manual driving), and thereby increasing freeway capacity by the same factor. A group of vehicles traveling with short headway is called a platoon. The earliest demonstration of an 8-car platoon of vehicles traveling 16 feet apart at 60 mph, was conducted on the I-15 freeway in San Diego in August 1997 ([1, 2]). Several other demonstrations have been carried out since then, e.g. $([3,4])$. These demonstrations show that headway reduction by a factor of 2 to 3 can be achieved today.

However, increasing the capacity of urban roads by platooning would not increase the throughput of an urban road network whose bottlenecks occur at intersections. For example, In a standard intersection with 4 approaches and two lanes-one through and one left-turn lane each with a capacity of 2000

** This research is funded by California Department of Transportation UCTC Award 65A0529 TO041. $\operatorname{vph}($ vehicles per hour) -the total capacity of the roads leading to the intersection is $8 \times 2000=$ $16,000 \mathrm{vph}$. But the intersection can accommodate only two movements safely at the same time, hence the intersection's capacity is only 4,000 vph or 25 percent of the road capacity. So increasing the latter capacity by platooning will do nothing to increase the capacity of the road network.

This observation is at the core of the remarkable study [5] to increase the throughput or saturation flow rate of an intersection by having cars cross the intersection in a platoon of ACC or CACC vehicles. The study concludes: if the saturation flow rates at all intersections in an urban network is increased by a factor $\Gamma$, "the network can support an increase in demand by the same factor $\Gamma$, with no increase in queuing delay or travel time, and using the same signal control. However, the queues will also grow by the same factor $\Gamma$, so if this leads to a saturation of the links, the improvement in throughput will be sub-linear in $\Gamma$. On the other hand, if the cycle time is reduced, the queues will also be reduced, and this may restore the linear growth in demand."

The authors stress two limitations of their study, which this paper overcomes. First, the study assumes that there is a 100 percent penetration of (C)ACC vehicles, whereas the present paper studies the effect of an arbitrary proportion of manual, $\mathrm{CC}$ and ACC vehicles. (The effect on freeway capacity of such arbitrary proportions is evaluated in [6].) "The second limitation is that in short urban links vehicles will slow down quickly as queues build up. As a result the saturation flow rate at the upstream intersection will be reduced, thereby depriving the system of the full productivity benefit. It is important to investigate this reduction." That investigation is carried out in this paper.

Our investigation is carried out on the basis of simulaton results using the Improved Intelligent Driver Model [7] or IIDM in SUMO. IIDM improves upon the IDM model [8] by eliminating some of the latter's unrealistic behaviors. IIDM is reviewed in Section 2. 
In Section 3 we discuss intersection throughput for manually driven vehicles, and in Section 4, we investigate the increase in throughput due to (C)ACC. In Section 5 we introduce the platoon model and evaluate $\mathrm{ACC}$ and $\mathrm{CACC}$ in terms of travel time and network throughput using SUMO simulation [9] of the 4-mile stretch of Colorado Boulevard / Huntington Drive arterial with 13 signalized intersections in Arcadia, Southern California. Section 6 concludes the paper.

\section{Car Following Model}

We start with the notation in Table 1 , and the default parameter values that we will use. The state equations for the IIDM car-following model are:

$$
\begin{aligned}
v(t+\Delta t) & =v(t)+a(t) \Delta t \\
x(t+\Delta t) & =x(t)+v(t) \Delta t+\frac{a(t) \Delta t^{2}}{2} .
\end{aligned}
$$

The critical control variable is acceleration $a(t)$, set by

$$
\begin{aligned}
& a(t)= \\
& \left\{\begin{array}{l}
a_{\max }\left(1-\left(\frac{g_{d}(t)}{g(t)}\right)^{\delta_{1}}\right), \text { if } \frac{g_{d}(t)}{g(t)}>1 \\
a^{*}(t)\left(1-\left(\frac{g_{d}(t)}{g(t)}\right)^{\frac{\delta_{1} a_{\max }}{a^{*}(t)}}\right), \text { otherwise }
\end{array}\right.
\end{aligned}
$$

where

$$
\begin{aligned}
a^{*}(t)= & a_{\max }\left(1-\left(\frac{v(t)}{v_{\max }}\right)^{\delta_{2}}\right), \\
g_{d}(t)= & g_{\min }+\max \{0, \\
& \left.v(t) \tau+\frac{v(t)\left(v(t)-v_{l}(t)\right)}{2 \sqrt{a_{\max } b}}\right\},
\end{aligned}
$$

and $\delta_{1}, \delta_{2}$ are fixed positive parameters. We use $\delta_{1}=$ $4, \delta_{2}=8$. The equilibrium speed $(a(t)=0)$ is given by $v(t)=v_{l}(t)=v_{\max }$ and $g(t)=g_{\min }+v(t) \tau$, and then the equilibrium headway is

$$
\theta_{e}=\tau+\frac{g_{\min }+l}{v_{\max }} .
$$

\begin{tabular}{|c|c|c|}
\hline Symbol & Description & $\begin{array}{l}\text { Default } \\
\text { value }\end{array}$ \\
\hline$l$ & Vehicle length. & $5 \mathrm{~m}$ \\
\hline$t$ & Time. & \\
\hline$\Delta t$ & Model time step. & $0.05 \mathrm{~s}$ \\
\hline$x(t)$ & Vehicle position. & \\
\hline$x_{l}(t)$ & Position of the lead vehicle. & \\
\hline$v_{\max }$ & Speed limit. & $20 \mathrm{~m} / \mathrm{s}$ \\
\hline$v(t)$ & Vehicle speed. & \\
\hline$v_{l}(t)$ & Speed of the leader. & \\
\hline$a(t)$ & Vehicle acceleration. & \\
\hline$a_{\max }$ & Maximal vehicle acceleration. & $1.5 \mathrm{~m} / \mathrm{s}^{2}$ \\
\hline$b$ & $\begin{array}{l}\text { Desired vehicle deceleration. } \\
\text { Gap: distance from the front }\end{array}$ & $2 \mathrm{~m} / \mathrm{s}^{2}$ \\
\hline$g(t)$ & $\begin{array}{l}\text { of the vehicle to the tail of the } \\
\text { leader, } g(t)=x_{l}(t)-x(t)-l \text {. }\end{array}$ & \\
\hline$g_{\min }$ & Minimal admissible gap. & $4 \mathrm{~m}$ \\
\hline$g_{d}(t)$ & Desired gap. & \\
\hline$\tau$ & Reaction time. & $2.05 \mathrm{~s}$ \\
\hline$\theta(t)$ & Headway: $\theta(t)=\frac{x_{l}(t)-x(t)}{v(t)}$ & \\
\hline$f(t)$ & Vehicle flow: $f(t)=\frac{1}{\theta(t)}$. & \\
\hline
\end{tabular}

The default values from Table 1 give $\theta_{e}=2.5 \mathrm{~s}$, which translates to $f_{e}=1 / \theta_{e}=0.4$ vehicles per second, 24 vehicles per minute, or $1440 \mathrm{vph}$. Empirical estimates of throughput vary between 1200 and 1900 vph.

\section{Intersection Throughput}

We analyze the urban arterial of Fig. 1 with infinitely many vehicles in the queue with minimal gap between
Table 1: Notation summary.

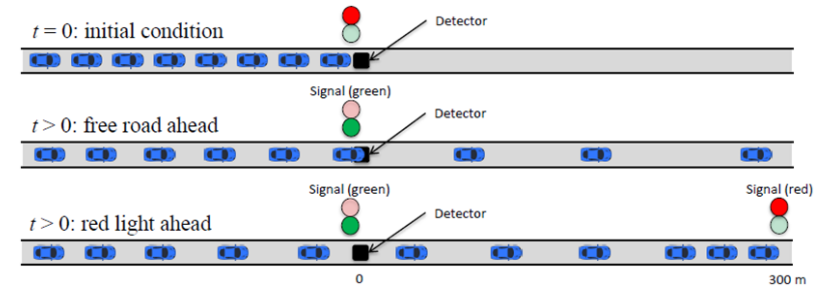

Figure 1: Signal turns green at time $t=0$, and vehicles start moving. In the first experiment, the first vehicle has free road ahead. In the second experiment, the first vehicle encounters red light at the next intersection, 300 meters downstream.

them. At time $t=0$, the light turns green, and vehicles are released. We discuss two experiments - one with free road ahead, and the second with a red light $300 \mathrm{~m}$ downstream at the second intersection. This distance accommodates 33 vehicles in the queue (see Table 1 for default values of the car length $l$ and the minimal gap $g_{\mathrm{min}}$ ), which exceeds the number that can be sent from the first signal in one minute.

Fig. 2 displays trajectories, speeds and accelerations of the first ten vehicles from the queue for these two experiments. The signal is located at position 0 and indicated by the horizontal black line in the top three trajectory plots. The leader of the first vehicle is infinitely far ahead. In the experiment with free road downstream, it accelerates with $a^{*}(t)$ from equation (4), asymptotically approaching the maximum 

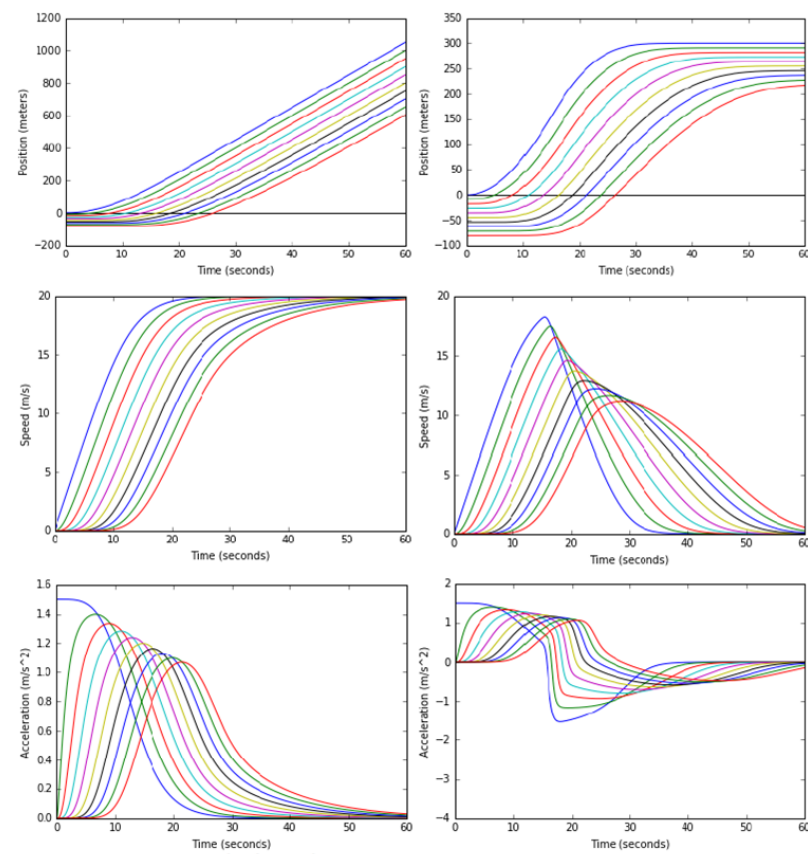

Free road ahead

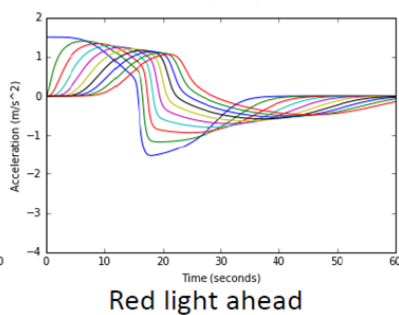

Figure 2: Vehicle trajectories, speeds and accelerations: experiment with free road ahead (left); and experiment with red light ahead (right).

speed. In the second experiment with the red light at position $x_{s}=300$, a "blocking vehicle" is placed at position $x_{b}=x_{s}+g_{\min }+l=300+4+5=309$, with velocity $v_{b}=0$, which causes the first vehicle to stop at $x_{s}$ to maintain the minimal gap.

As a queue builds up in front of the red light, vehicles slow down, and thereby reduce the vehicle flow though the first intersection. In the experiment with the red light downstream we study the impact of this braking effect on the throughput of the first intersection. (This is the second limitation noted in [5].)

The most interesting for the intersection throughput assessment is the traffic behavior at the stop bar location indicated in Fig. 1. Fig. 3 presents the measurements obtained from this detector, with each dot corresponding to a vehicle passing the detector. The (instantaneous) flow (top left) is computed for a vehicle passing the detector based on the time elapsed after the previously detected vehicle, taken as a headway $\theta(t)$, which is then inverted $(f(t)=1 / \theta(t))$ and expressed in vph. The red horizontal line corresponds to the equilibrium flow of $1440 \mathrm{vph}$ in our case, when vehicles move at maximal speed. The gap (top right) between vehicles as well as the speed (bottom left) are monotonically increasing, while acceleration (bottom right) is monotonically decreasing.

In the case of free road downstream, 23 vehicles cross the intersection during the first minute, slightly be-
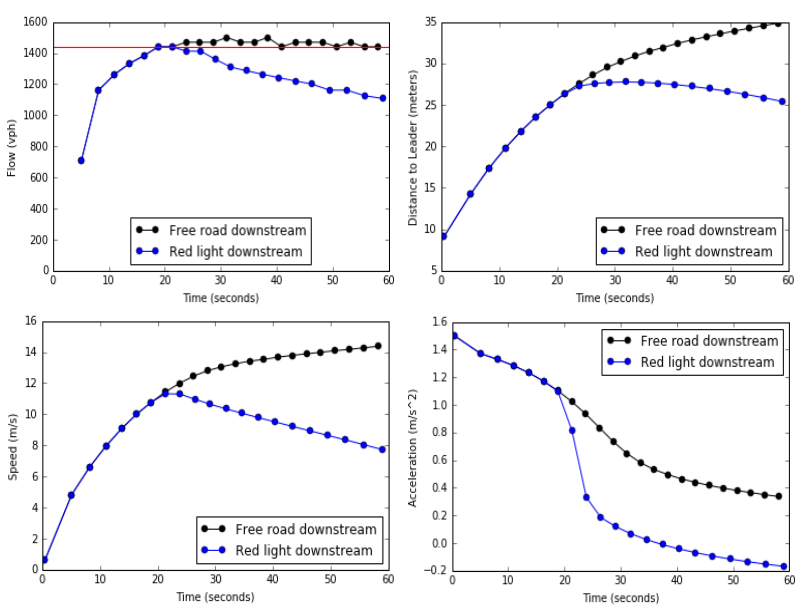

Figure 3: Experiment with a free road ahead: comparison of measurements of flow, distance to leader, speed and acceleration at the detector location between the three car following models.

low the equilibrium flow of 24 vehicles per minute. The IIDM control can be tuned to a more aggressive behavior by increasing its parameters $\delta_{1}, \delta_{2}$. In the case of red light downstream, the flow is reduced to 21 vehicles during the first minute as the result of braking propagation.

We now examine how throughput at the first intersection in our two previous experiments depends on the maximal acceleration $a_{\max }$. We repeat both experiments, with the free road and with the red light ahead, for three different values of $a_{\max }: 0.8,1.5$ (our default) and $2.5 \mathrm{~m} / \mathrm{s}^{2}$. Fig. 4 presents the measurements obtained at the stop bar location for the cases of free road (left) and red light downstream (right). Table 2 summarizes the throughput results for different acceleration values. The results for this 'base' case will be compared with the increased throughput using (C)ACC.
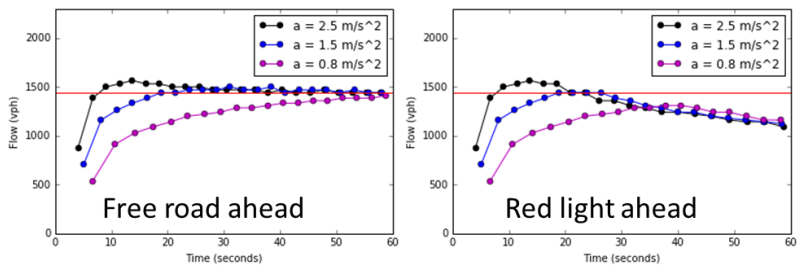

Figure 4: Comparing flows for different values of $a_{\max }$ for experiments with the free road (left), and with the red light ahead (right).

\section{Effect of ACC and CACC}

We will now explore the impact of ACC and CACC vehicles on intersection throughput. To do that, we 


\begin{tabular}{|c|c|c|}
\hline Value of $a_{\max }$ & Type of experiment & IIDM \\
\hline \multirow{2}{*}{$0.8 \mathrm{~m} / \mathrm{s}^{2}$} & free road ahead & 20 \\
& red light ahead & 19 \\
\hline \multirow{2}{*}{$1.5 \mathrm{~m} / \mathrm{s}^{2}$} & free road ahead & 23 \\
& red light ahead & 21 \\
\hline \multirow{2}{*}{$2.5 \mathrm{~m} / \mathrm{s}^{2}$} & free road ahead & $\mathbf{2 4}$ \\
& red light ahead & 22 \\
\hline
\end{tabular}

Table 2: Summary of experiments: intersection throughput in vehicles per minute. Values equal to the equilibrium flow are in bold.

repeat two experiments described in Section 3 but this time, with a mix of ordinary (manually driven), ACC and CACC vehicles. Values of car following parameters for the three vehicle types are given in Table 3. As one can see, ACC and CACC vehicles can maintain shorter distances to the vehicle in front.

\begin{tabular}{|l|c|c|}
\hline Vehicle type & $\tau$ (seconds) & $g_{\min }$ (meters) \\
\hline Ordinary & 2.05 & 4 \\
ACC-enabled & 1.1 & 3 \\
CACC-enabled & 0.8 & 3 \\
\hline
\end{tabular}

Table 3: Values of reaction time $\tau$ and minimal gap $g_{\text {min }}$ for ordinary, ACC- and CACC-enabled vehicles.

We assume that the ACC vehicle has the same car following model as that of the ordinary vehicle, but with different $\tau$ and $g_{\min }$. A CACC vehicle behaves like an $\mathrm{ACC}$ vehicle if it follows an ordinary vehicle, but if it has another CACC car in front, it assumes different car following behavior, which we call $C A C C$ car-following model.

Let $a_{I I D M}(t)$ denote the acceleration function defined by (3). Define the constant-acceleration heuristic $(C A H)$ acceleration function [7]:

$$
\begin{aligned}
& a_{C A H}(t)= \\
& \left\{\begin{array}{l}
\frac{v^{2}(t) \bar{a}_{l}(t)}{v_{l}^{2}(t)-2\left(x_{l}(t)-x(t)-l\right) \bar{a}_{l}(t)} \\
\text { if } \frac{v_{l}(t)\left(v(t)-v_{l}(t)\right)}{2 \bar{a}_{l}(t)} \leq\left(x(t)+l-x_{l}(t)\right) ; \\
\bar{a}_{l}(t)-\frac{\left(v(t)-v_{l}(t)\right)^{2} \Theta\left(v(t)-v_{l}(t)\right)}{2\left(x_{l}(t)-x(t)-l\right)}, \text { otherwise, }
\end{array}\right.
\end{aligned}
$$

where

$$
\bar{a}_{l}(t)=\min \left\{\dot{v}_{l}(t), a_{\max }\right\},
$$

and

$$
\Theta(z)= \begin{cases}1, & \text { if } z \geq 0 \\ 0, & \text { otherwise }\end{cases}
$$

We now specify the CACC car following model [7]:

$$
\begin{aligned}
& a_{C A C C}(t)= \\
& \left\{\begin{array}{l}
a_{I I D M}(t), \text { if } a_{C A H}(t) \leq a_{I I D M}(t), \\
a_{C A H}(t)+ \\
b \tanh \left(\frac{a_{I I D M}(t)-a_{C A H}(t)}{b}\right), \text { otherwise. }
\end{array}\right.
\end{aligned}
$$

As before, we run the free road and the red light downstream experiments. For both scenarios we compute the intersection throughput, when portion of ACC (CACC) vehicles in the initial queue. Thus we evaluate 24 cases, each defined by: (1) experiment: free road or red light downstream; (2) ACC or CACC; and (3) percentage of ACC (CACC): 10, 25, 50, 75,90 and $100 \%$.

Fig. 5 and 6 compare flows, gaps, speeds and acceleration obtained at the detector location for 0,50 and $100 \%$ ACC (CACC) penetration rate. Three horizontal red lines on flow plots in both figures correspond to equilibrium flows with $0 \%$ ACC (CACC), with $100 \%$ ACC and with $100 \%$ CACC penetration rate. These flows are computed as $3600 / \theta_{e}$, where $\theta_{e}$ is given by (6) with $\tau$ and $g_{\min }$ from Table 3 , yielding 1440,2400 and 3000 vehicles per hour respectively. In the flow and distance to leader plots, notice how $50 \%$ ACC plots jump between the no ACC and $100 \%$ ACC plots: for an ordinary vehicles it is similar to the no ACC curve, and for an ACC vehicle it is similar to $100 \%$ ACC curve. $50 \%$ CACC curves in the same plots jump between three curves - no ACC, $100 \% \mathrm{ACC}$ and $10 \% \mathrm{CACC}$. This is because a CACC vehicle following an ordinary one behaves like ACC vehicle.
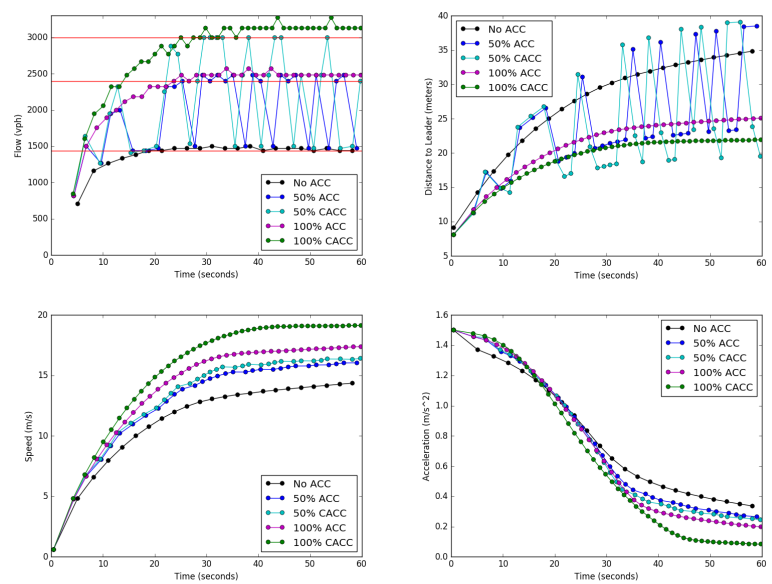

Figure 5: Case with free road ahead: comparison of measurements of flow, distance to leader, speed, and acceleration at the detector location for different proportions of ACC/CACC traffic. 

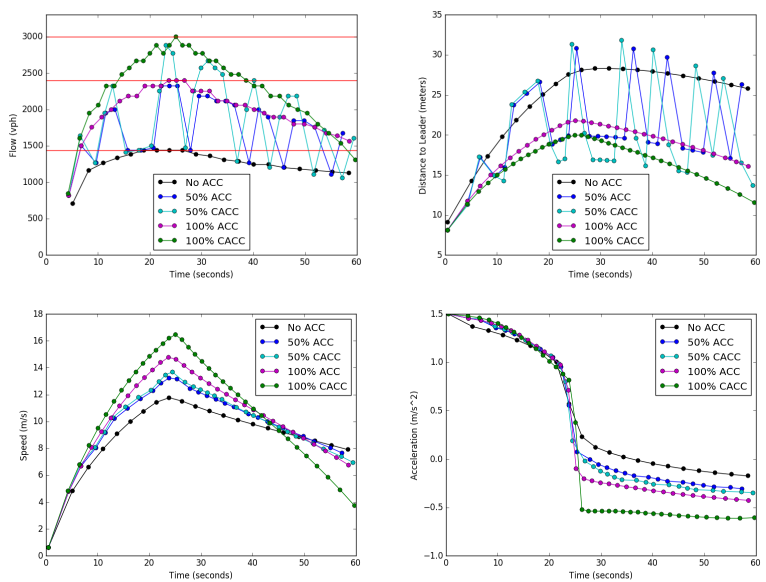

Figure 6: Case with red light downstream: comparison of point measurements of flow, distance to leader, speed and acceleration at the detector location for different proportions of $\mathrm{ACC} / \mathrm{CACC}$ traffic.

For an ACC (CACC) penetration rate less than $100 \%$, the intersection throughput is sensitive to the distribution of ACC (CACC) vehicles in the initial queue. For example, if $25 \%$ all vehicles in the initial queue are ACC-enabled, and all of them are concentrated at the head of the queue, we would get higher vehicle count at the detector location after one minute, than we would with $50 \%$ ACC penetration rate when all ACC-enabled vehicles are concentrated at the tail of the queue. In another example, $50 \%$ CACC penetration rate would not produce any gain over $50 \%$ ACC penetration rate, if ordinary and $\mathrm{ACC} / \mathrm{CACC}$ vehicles were interleaved - one ordinary, one $\mathrm{ACC} / \mathrm{CACC}$, one ordinary, and so on - since an CACC achieves a shorter headway than ACC only when it has other CACC vehicles directly in front.

To mitigate this ACC (CACC) distribution bias, in each case with ACC (CACC) penetration rate less than $100 \%$, we run 100 random one-minute simulations of the three car-following models, record vehicle counts at the detector location, and plot the median vehicle count. For $100 \%$ penetration rate the ACC (CACC) distribution is trivial, and hence, a single simulation for each case is enough. The intersection throughput results for all the 24 cases, together with throughput values from Table 2 obtained for 0 ACC (CACC) penetration rate, are presented as the four plots in Fig. 7.

Note that in each of the four plots in Fig. 7, there is a curve corresponding to the equilibrium traffic flow. These equilibrium curves are computed as follows. Denote by $\lambda \in[0,1]$ the fraction of ACC (CACC) vehicles in the initial queue, and by $\tau^{[C] A C C}$ and
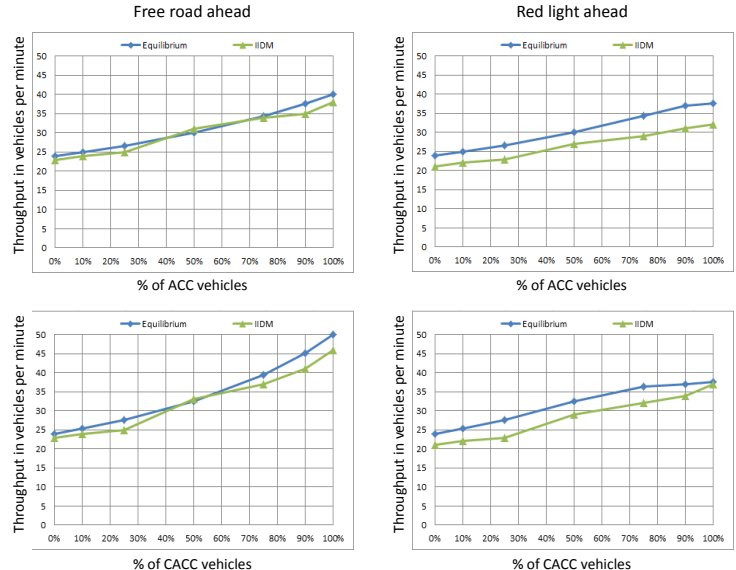

Figure 7: Intersection throughput as a function ACC (top) or CACC (bottom) portion of traffic computed with IIDM for free road downstream (left) and red light downstream (right).

$g_{\min }^{[C] A C C}$ the reaction time and minimal gap for ACC (CACC) vehicles, whose values are given in Table 3. The average headway in the equilibrium state is obtained by modifying expression (6):

$$
\begin{aligned}
\theta(\lambda)= & \lambda \tau^{[C] A C C}+(1-\lambda) \tau+ \\
& \frac{\lambda g_{\min }^{[C] A C C}+(1-\lambda) g_{\min }+l}{v_{\max }} .
\end{aligned}
$$

Then, the equilibrium flow in vehicles per minute is given by:

$$
f(\lambda)=60 / \theta(\lambda) .
$$

This formula is sufficient for the case when there is a free road ahead. In the case of a red light downstream, however, we are restricted by the capacity of the link connecting the two intersections. To account for that, we modify (10) accordingly:

$$
\begin{aligned}
f(\lambda)= & \min \left\{\frac{60}{\theta(\lambda)},\right. \\
& \left.\frac{k \Delta}{\lambda g_{\min }^{[C] A C C}+(1-\lambda) g_{\min }+l}\right\},
\end{aligned}
$$

where $\Delta$ is the length of the link between the two intersections, and $k$ is the number of lanes in that link. In our experiment, $\Delta=300$, and $k=1$.

\section{Platoons on Road Network}

Vehicles equipped with CACC can form platoons. With 50\% CACC penetration rate, platoons provide between 24 and $44 \%$ increase in intersection throughput on average, depending on the proximity of intersections. 
In simulation, platoon management and formation is divided into three phases: 1) Identifying vehicles that can be grouped into platoons; 2) Adjusting parameters of leaders and followers in platoons; 3) performing maintenance on the platoon. This behavior is modeled by the state machine in Fig. 8 .

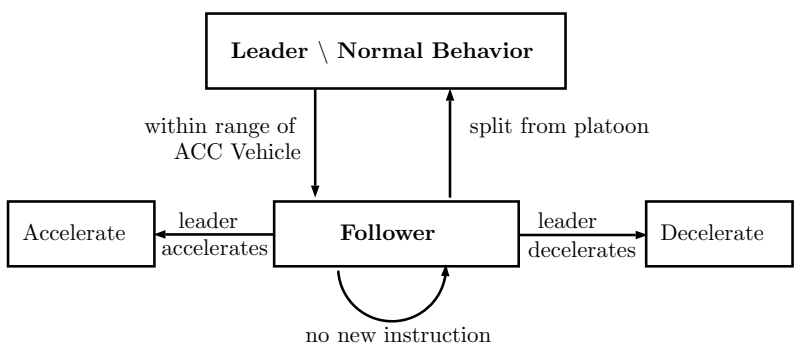

Figure 8: State machine of a platoon vehicle.

To form a platoon, vehicles must be in sequence with one another on a given lane. However, vehicles need not share the same final destination and are free to switch lanes or leave the platoon if necessary. If an intermediate vehicle in the platoon changes its route by making a turn or changing lanes, the platoon splits into two: one platoon for the vehicles ahead of the intermediate vehicle and another for all the vehicles behind.

A platoon's lead vehicle has the same properties as ACC vehicles. An isolated CACC vehicle is a leader of a platoon of size 1 . When a platoon leader comes into range of another $\mathrm{CACC}$ vehicle in front, it joins the platoon becoming a follower. Followers have reduced headway and travel much closer to one another than standalone vehicles. In addition, followers are able to receive information from the leader, such as to accelerate after a green light at an intersection or to decelerate approaching an obstacle, e.g. red light, downstream.

Since followers are not bound to the same route as the platoon leader, they are free to separate. After leaving the platoon, the headway and acceleration parameters are restored to their original values. This can happen for example when the follower changes its route or becomes separated from the rest of platoon, e.g., due to switching traffic signal as it crosses the intersection.

To study the impact of platooning, we used a SUMO model of the 4-mile stretch of Colorado Boulevard / Huntington Drive arterial with 13 signalized intersections in Arcadia, Southern California, shown in Fig. 9. IIDM and CACC models were implemented in SUMO, and platoon management and formation were handled via SUMO/TraCI API. Using real world flow measurements and estimated turn ratios at in- tersections, we generated 1 hour of origin-destination (OD) travel demand data. Then, we ran a series of

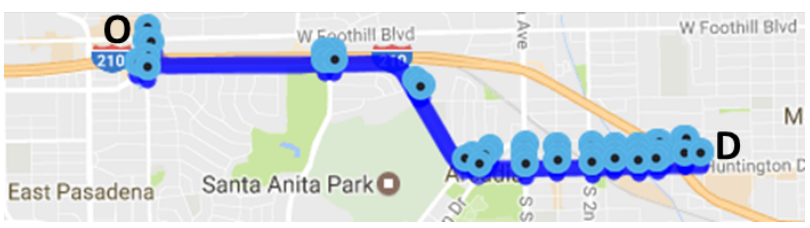

Figure 9: Huntington Colorado network modeled in SUMO.

simulation varying the fraction of ACC (CACC) vehicles from 0 to $75 \%$. In each simulation two vehicle classes were modeled: ordinary vehicles and ACC (or CACC) vehicles. In simulations with CACC vehicles platoons were formed. The total number of OD pairs in this network is 399 . The same number of vehicles was processed in each simulation. The rates and locations at which cars were generated were identical in all scenarios to eliminate the variance in randomly generated routes. For cases of $0,25,50$ and 75 percent $\mathrm{ACC}$ (CACC) penetration rate, we computed average travel time for the route $\mathrm{O} \rightarrow \mathrm{D}$, where $\mathrm{O}$ and $\mathrm{D}$ identify origin and destination of the selected westeast route in Fig. 9. Table 4 lists the mean travel time (MTT) and its standard deviation (STD), in seconds. As expected, the mean travel time reduces as the fraction of (C)ACC vehicles increases. Surprisingly the standard deviation also decreases. Furthermore, the travel time of ordinary vehicles is also reduced, although that of (C)ACC vehicles is reduced more.

\begin{tabular}{|c|c|c|c|c|c|}
\hline [C]ACC & Vehicle & \multicolumn{2}{|c|}{ ACC } & \multicolumn{2}{c|}{ CACC } \\
\cline { 3 - 6 }$\%$ & Class & MTT & STD & MTT & STD \\
\hline 0 & ordinary & 653 & 102 & 653 & 102 \\
\hline \multirow{3}{*}{$25 \%$} & ordinary & 640 & 96 & 638 & 96 \\
& [C]ACC & 605 & 82 & 600 & 76 \\
& all & 631 & 94 & 629 & 94 \\
\hline \multirow{3}{*}{$50 \%$} & ordinary & 583 & 66 & 579 & 60 \\
& [C]ACC & 583 & 61 & 570 & 64 \\
& all & 583 & 64 & 575 & 62 \\
\hline \multirow{2}{*}{$75 \%$} & ordinary & 595 & 45 & 583 & 41 \\
& [C]ACC & 558 & 58 & 540 & 52 \\
& all & 567 & 57 & 550 & 48 \\
\hline
\end{tabular}

Table 4: Mean travel time (MTT) and standard deviation (STD) in seconds for varying percentage of ACC vehicles on the main arterial of Fig. 9.

\section{Conclusion}

Presence of (C)ACC-enabled vehicles in the traffic increases the intersection throughput and reduces 
travel time at all levels of penetration. CACC vehicles forming a sequence increase the throughput of an intersection significantly more than can be achieved with pure ACC vehicles; but CACC vehicles interleaved with ordinary ones have the same effect as ACC vehicles. Queues in short links do reduce the throughput at an upstream intersection.

In an urban network the presence of $(\mathrm{C}) \mathrm{ACC}$ vehicles reduces the queues and hence the time spent at intersections. As a result ordinary as well as (C)ACC vehicles benefit from lower average travel times with a lower standard deviation. The results of this paper together with those in [5] indicate that (C)ACC can greatly improve urban road mobility at little or no additional infrastructure cost.

\section{References}

[1] NAHSRC, "Automated Highway Demo 97," https: / /www.youtube.com/watch?v= C9G6JRUmg_A.

[2] S. Shladover, "Why automated vehicles need to be connected vehicles," 2013, http://www.ewh.ieee.org/tc/its/VNC13/IEEE VNC_BostonKeynote_Shladover.pdf.

[3] J. Ploeg, B. T. M. Scheepers, E. van Nunen, N. van de Wouw, and H. Nijmeijer, "Design and experimental evaluation of cooperative adaptive cruise control," Proc. 14th ITSC IEEE Conf, pp. 260-265, 2011.

[4] V. Milanes, S. E. Shladover, J. Spring, C. Nowakowski, H. Kawazoe, and M. Nakamura, "Cooperative adaptive cruise control in real traffic situations," IEEE Trans. Intelligent Transportation Systems, vol. 15, no. 1, pp. 296-305, Feb 2014.

[5] J. Lioris, R. Pedarsani, F. Tascikaraoglu, and P. Varaiya, "Platoons of connected vehicles can double throughput in urban roads," Transportation Research, Part C, to appear.

[6] H. S. Mahmassani, "50th anniversary invited articleâǍTautonomous vehicles and connected vehicle systems: Flow and operations considerations," Transportation Science, 2016, published online.

[7] M. Treiber and A. Kesting, Traffic Flow Dynamics: Data, Models and Simulation. Springer, 2013.

[8] M. Treiber, A. Hennecke, and D. Helbing, "Congested traffic states in empirical observations and microscopic simulations," Physical Review E, vol. 62, no. 2, pp. 1805-1824, 2000.

[9] D. Krajzewicz, "Traffic simulation with SUMOsimulation of urban mobility," in Fundamentals of Traffic Simulation. Springer, 2010, pp. 269293, http://www.dlr.de/ts/en/desktopdefault. aspx/tabid-9883/16931_read-41000, accessed $04 / 28 / 2016$. 